\title{
Enfermedad arterial periférica y pié diabético en pacientes en programa de hemodiálisis
}

\author{
Azucena García Viejo
}

Graduada en enfermería. Unidad de diálisis Hospital General de Segovia

\section{Resumen}

A pesar de la alta prevalencia de la enfermedad cardiovascular, y diabetes en los pacientes en diálisis, no existen muchos estudios que analicen la incidencia de los factores de riesgo y las repercusiones de la arteriopatía periférica y pie diabético en pacientes en diálisis.

\section{Objetivos:}

Determinar la prevalencia de la arteriopatía periférica y pie diabético en los pacientes en diálisis de nuestra unidad.

Realizar una clasificación por riesgo que nos permita un mejor seguimiento y prevención de las ulceras y amputaciones en miembros inferiores.

\section{Material y métodos}

Estudio observacional descriptivo y transversal. Realizado con 51 pacientes incluidos en programa de hemodiálisis en la Unidad de Segovia. Se realizaron análisis de los diferentes factores de riesgo que influyen en la presencia de arteriopatía y pie diabético, y las diferentes relaciones entre ellos así como una estratificación del riesgo en la población estudiada.

\section{Resultados}

Un $45 \%$ de los pacientes presenta arteriopatía periférica y un $58 \%$ tiene alteraciones de la sensibilidad. La diabetes en primer lugar y las enfermedades car-

\section{Correspondencia: \\ Azucena García Viejo \\ Carretera de Villacastín $225^{\circ} \mathrm{A}$ \\ 40006. Segovia \\ e-mail: agarciav60@hotmail.com}

diovasculares son los factores que más influyen en estos problemas.

\section{Conclusiones}

La categorización de riesgo de nuestros pacientes permitirá planificar las estrategias para prevenir la aparición de estas complicaciones. La ausencia de exploración anterior muestra un seguimiento inadecuado. La alta prevalencia de estas patologías hace necesario un seguimiento protocolizado.

\section{PALABRAS CLAVE: \\ - ARTERIOPATIA PERIFÉRICA \\ - DIABETES MELLITUS \\ - HEMODIÁLISIS \\ - NEUROPATÍA DIABÉTICA \\ - ÍNDICE TOBILLO BRAZO \\ - MONOFILAMENTO SEMMES-WEINSTEIN}

\section{Peripheral arterial disease and diabetic foot in patients on haemodialysis programme}

\section{Abstract \\ Despite the high prevalence of cardiovascular disease and diabetes in patients on dialysis, there are not many studies that analyse the incidence of the risk factors and the repercussions of peripheral arteriopathy and diabetic foot in patients undergoing dialysis.}

\section{Aims}

To determine the prevalence of peripheral arteriopathy and diabetic foot in dialysis patients in our unit. 
To establish a classification by risk that will allow us to carry out a better monitoring and prevention of ulcers and amputations of lower limbs.

\section{Material and methods}

Transversal descriptive observational study. Carried out with 51 patients included in the haemodialysis programme of the Unit in Segovia. Analyses were carried out of the different risk factors that influence the presence of arteriopathy and diabetic foot, and the different relationships between them as well as a stratification of the risk in the population studied.

\section{Results}

$45 \%$ of the patients present peripheral arterial disease and $58 \%$ have sensitivity alterations. Diabetes firstly and cardiovascular diseases are the factors that have the most influence in these problems.

\section{Conclusions}

Categorizing our patients according to risk will make it possible to plan strategies to prevent the appearance of these complications. The absence of previous examination shows inadequate monitoring. The high prevalence of these pathologies makes protocolized monitoring necessary.

\section{KEY WORDS}

- PERIPHERAL ARTERIAL DISEASE
- DIABETES MELLITUS
- HAEMODIALYSIS
- DIABETIC NEUROPATHY
- ANKLE-BRACHIAL INDEX
- SEMMES-WEINSTEIN MONOFILAMENT

\section{Introducción}

La prevalencia de la enfermedad cardiovascular en pacientes en diálisis es muy alta, entre el 40 y el $75 \%$ de los pacientes que comienzan diálisis tienen enfermedad cardiovascular... Este tipo de pacientes tiene mas riesgo cardiovascular que la población general y esto es debido fundamentalmente a que en los pacientes en diálisis se superponen factores clásicos (HTA, obesidad, hiperlipemia y tabaco), los factores específicos de la uremia (hiperparatiroidismo) y los factores relacionados con la diálisis ${ }^{(1)}$.

Entre las alteraciones cardiovasculares más importantes en estos pacientes tenemos: la hipertrofia del ventrículo izquierdo, la enfermedad coronaria, las calcificaciones vasculares y la arteriopatía o enfermedad oclusiva periférica (principal causa de amputación de extremidades y supone un alto riesgo de morbilidad y mortalidad en diálisis).

La prolongación de la esperanza de vida y el aumento de la prevalencia de la insuficiencia renal crónica, secundaria a nefropatía diabética y etiología vascular hacen que asistamos desde hace años a la entrada en programa de tratamiento sustitutivo de pacientes sin límite de edad y con patología asociada a riesgo cardiovascular. Esta situación se asocia a un aumento importante de la morbilidad de origen cardiovascular y en el caso de la arteriopatía periférica se ha observado que el aumento de esta patología entre los pacientes en diálisis, incrementa el riesgo de muerte de 3 a 6 veces en las formas asintomáticos y hasta 15 veces en las formas severas ${ }^{(2)}$.

La presencia de arteriopatía periférica puede inhibir gravemente o impedir la capacidad de una extremidad inferior para curar ulceras. Hay un buen número de heridas en las extremidades inferiores que no se curaran mientras no se restablecta una perfusión adecuada(3).

Además es importante destacar que la diabetes es la enfermedad que mas se asocia a la enfermedad arterial periférica lo que implica un gran numero de lesiones vasculares en miembros inferiores y la presencia de complicaciones crónicas como el pié diabético.

Esta patología tiene una prevalencia que oscilaría entre el $1,5 \%$ y el $10 \%$, que correspondería a una incidencia entre el $2,2 \%$ y el $5,9 \%$, lo que se traduce en que, cada año, unos cuatro millones de diabéticos desarrollan una úlcera en el pié.

Se ha demostrado que una prevención adecuada de las lesiones en el pie del diabético, así como un correcto tratamiento puede reducir esta tasa de amputación entre un 50 y un $85 \%$. $^{(4)}$

Los costes económicos ocasionados por estas complicaciones para los sistemas sanitarios son enormes, así como 
las repercusiones sociales y emocionales que provoca , lo que hace imprescindible el estudio mas amplio de su prevalencia en los pacientes en diálisis y la puesta en marcha de sistemas para prevenir estas patologías. La prevención tendría como objetivo definir, proponer, consensuar y realizar una serie de acciones estratégicas con la finalidad de evitar la aparición de lesiones.

Una estrategia válida para ello, sería la implantación de planes de cuidados estandarizados para establecer y llevar a cabo las intervenciones necesarias encaminadas a resolver este problema de salud. Un paso previo para la puesta en marcha de estos planes, seria la identificación de pacientes en nuestra Unidad de Hemodiálisis, con perfusión periférica alterada ya sea por diabetes o cualquier otro tipo de alteración vascular.

Esta tipificación, nos permitirá conocer los pacientes de riesgo para amoldar los planes de prevención y cuidados al riesgo que detectemos en nuestros pacientes, lo que nos proporcionaría decidir las estrategias más adecuadas para prevenir y tratar las lesiones y evitar gangrenas y amputaciones.

La presencia de enfermería ante este planteamiento se justifica en la necesidad de detectar que factores de riesgo son los que con mayor frecuencia aparecen en nuestro entorno. El diagnostico de la alteración en la perfusión de miembros inferiores se realiza mediante una angiografía, pero según ciertos autores esta prueba solo debería realizarse en pacientes candidatos a revascularización mecánica o quirúrgica. Las indicaciones de angioplastia en diálisis están muy limitadas por la alta incidencia de lesiones dístales y de calcificaciones vasculares ${ }^{(5)}$. Existen otros tipos de exploraciones que nos pueden indicar la presencia de arteriopatía periférica sin utilizar métodos invasivos ${ }^{(6)}$. El índice tobillo- brazo, la presencia de pulsos y la exploración de sensibilidad, etc. indicaran riesgo de lesión en los miembros inferiores de pacientes en diálisis.

Para la prevención, detección precoz y tratamiento de estos problemas vasculares es fundamental que las enfermeras que cuidan a los pacientes en diálisis establezcan qué tipo de exploraciones se realizaran y con que frecuencia para evitar la aparición de lesiones y el resto de las complicaciones.

Las intervenciones de enfermería deben incluir la exploración del los miembros inferiores y del pié y constara de: inspección visual y hábitos higiénicos, exploración vascular y neuropática (exploración mediante el monofilamento de Semmes-Weinstein) y valoración de la capacidad de autocuidado.

Aunque no existe evidencia firme sobre la frecuencia en que estas revisiones deben hacerse, se acepta la realización de al menos un examen anual y con mayor frecuencia si existen factores de riesgo que así lo indiquen ${ }^{(4)}$.

A pesar del papel fundamental de la enfermería, las intervenciones enfermeras sobre prevención de las amputaciones y su registro son objetivos pendientes en los planes de cuidados de estos pacientes ${ }^{(7)}$.

Por tanto, la intervención de enfermería no debe limitarse a la exploración física y al registro de los datos relevantes, el propósito final debe ser iniciar medidas de educación en el paciente que conduzcan a una mejora de los diferentes factores de riesgo que presente cada paciente para evitar lesiones y amputaciones en los miembros inferiores.

A la vista del estado del problema nos planteamos los siguientes objetivos:

- Determinar la prevalencia de la arteriopatía periférica y pié diabético en los pacientes en diálisis de nuestra unidad.

- Realizar una clasificación por riesgo que nos permita un mejor seguimiento y prevención de las ulceras $\mathrm{y}$ amputaciones en miembros inferiores.

\section{Material y métodos}

Planteamos un estudio de tipo observacional descriptivo y transversal.

El tamaño de la muestra fue de 51 pacientes $(n=51)$, todos los pacientes incluidos en programa de hemodiálisis en la Unidad de Segovia.

El período de recogida de datos del presente estudio fue de 1 mes. Abril de 2011. Para llevar a cabo el estudio analizamos las siguientes variables independientes:

Sexo, edad, tiempo de permanencia en tratamiento sustitutivo, enfermedad de base. Diabetes, duración de la diabetes, enfermedad cardiovascular, presencia de pulsos, Índice tobillo brazo, ulcera o amputación previa. 
También se recogieron datos que indican riesgo de padecer lesiones como son: déficit de cuidados del pié, ambiente socioeconómico desfavorecido, disminución o perdida de sensibilidad, presencia de deformidades, presencia de helomas/ hiperqueratosis y calzado inadecuado.

Como variables dependientes tenemos: existencia de al menos un factor de riesgo y estratificación del riesgo.

\section{Definición de las variables:}

Diabetes: Se registra si el paciente es diabético o no.

Duración de la diabetes: menos de 10 años, mas de 10 años.

Presencia de enfermedad cardiovascular. Considerando que existe enfermedad cardiovascular si el paciente presenta: HTA, hipertrofia de ventrículo izquierdo o cardiopatía.

Pulsos presentes: Se consideraran sujetos con pulsos presentes a aquellas personas a las cuales se consiga palpar, al menos, el pulso pedio o el tibial posterior en ambos miembros inferiores.

Índice tobillo brazo (ITB): Método diagnostico no cruento utilizado para estimar la gravedad de la enfermedad vascular periférica o para establecer valores de referencia.EI valor normal de ITB oscila entre 0.9 y 1.3. Valores menores de 0.9 indican obstrucción en la extremidad y los > de 1.3-1.5 son también patológicos ya que indican perdida de la elasticidad vascular por calcificación arterial como ocurre en arteriopatía diabética o en la insuficiencia renal crónica. Un valor $<$ de 0.3 es sinónimo de isquemia critica(10) $^{(10)}$.

Déficit de cuidados de pié: Se entiende como déficit de cuidados relativos al pié los siguientes parámetros:

Personas que responden negativamente a la pregunta de si conoce los cuidados especiales que requieren sus pies, personas que niegan haber recibido nunca información específica relativa al cuidado de los pies, personas que no presentan una higiene adecuada en los pies el día de la exploración y personas que, tras inspección visual, se constata un corte de las uñas inadecuado, según las recomendaciones al respecto incluidas en el los documentos de consenso.
Aislamiento social/ambiente socioeconómico desfavorecido: Se considera la existencia de aislamiento social/ambiente socioeconómico desfavorecido en aquellos sujetos que, tras el acceso a su valoración de enfermería tenga registrado problemas de vulnerabilidad en lo referente a su situación familiar, problemas socioeconómicos y/o problemas afectivos graves ${ }^{(9)}$.

Úlcera/amputación previa: Se considera como úlcera previa a toda lesión en uno o ambos pies o piernas origen traumático o no traumático. Como amputación previa se entiende toda resección de alguna sección anatómica de una o ambas extremidades inferiores.

Disminución de la sensibilidad protectora: se considera que sufren disminución de sensibilidad protectora aquellos pacientes que tras exploración con monofilamento de Semmes-Weinstein 5,07 mm-10 g no perciben el estímulo en alguno de los puntos preestablecidos en uno o ambos pies en, al menos, 2 ocasiones tras tres intentos.

Deformidades del pié: Se considera como deformidades del pie a las siguientes alteraciones ${ }^{(9)}$ : Hallux valgus, pie plano, pie cavo y dedos en garra.

Helomas/hiperqueratosis: Presencia de zonas hiperqueratósicas tanto en zonas de presión como cabezas metatarsales, zonas dorsales de los dedos o espacios interdigitales como en otras zonas del pie como talones.

Calzado inadecuado: Se considera como calzado inadecuado el calzado que reúne, al menos, uno de los siguientes parámetros: ${ }^{(9)}$ Calzado en mal estado (roto, desgastado 0 deformado por el uso), calzado sin contrafuerte, calzado de punta estrecha que comprime los dedos, calzado con un tacón de altura superior a 2,5 cm calzado con costuras 0 imperfecciones en su interior. El calzado inspeccionado será el que lleve la persona en el momento de la visita para la entrevista/exploración.

Categorización del riesgo. Se utilizo la clasificación de riesgo del Documento de Consenso Internacional del Pie diabético del año $2007^{(10)}$ :

- Riesgo 1: Se incluyen en esta categoría a aquellas personas sujetas a estudio que no tengan ninguna historia de úlcera ni amputación previa, con pulsos presentes, sin deformidad y con sensibilidad protectora y profunda conservada. 
- Riesgo 2: Se incluyen en esta categoría a aquellas personas sujetas a estudio con pulsos presentes, pero donde se aprecie afectación de la sensibilidad protectora y/o profunda.

- Riesgo 3: Se incluyen en esta categoría a aquellas personas sujetas a estudio con la sensibilidad protectora y/o profunda afectada que presentaran, además, pulsos ausentes y/o con deformidad en los pies. También se incluyen en esta categoría aquellos sujetos con sensibilidad conservada, pero con pulsos ausentes ya que se interpretó la ausencia de pulsos como signo de enfermedad arterial periférica.

- Riesgo 4: Se incluyen en esta categoría a aquellas personas sujetas a estudio con historial previo de úlcera o amputación.

\section{Fuentes de datos}

El proceso de recogida de datos se realizo en tres partes:

Entrevista. Un interrogatorio a través de un formulario para identificar factores de riesgo y recoger información sobre determinadas variables (edad, diabetes y duración, enfermedad cardiovascular, conocimientos sobre autocuidados de los pies...).

Exploración física. Se efectuó una exploración individual en cada paciente que consistió en:

- Palpación manual del pulso pedio y tibial posterior de ambos pies. Comprobación del estado del pulso con Doppler portátil.

- Exploración de la sensibilidad táctil a la presión ligera (sensibilidad protectora) mediante el test del monofilamento según protocolo. Se utilizara el monofilamento Semmes-Weinstein 5,07 $\mathrm{mm}$. Con el fin de evitar desgaste del monofilamento por fatiga de material, se usara un nuevo monofilamento por cada 8 pacientes explorados.

- Inspección visual de ambos pies, donde se valorara la presencia de helomas o hiperqueratosis, de deformidades, corte de uñas, higiene del pie y la existencia de úlceras activas/lesiones.

- Inspección del calzado que lleva el sujeto en el momento de la entrevista-exploración, valorándose un número limitado de ítems indicados en el formulario de recogida de datos.

Este proceso se realizó en el ámbito físico de la unidad de hemodiálisis.

- Revisión de la historia clínica: tras la entrevista y la exploración se revisó la historia clínica de cada paciente con el fin de comprobar algunos aspectos como el registro de ulcera/amputación previa, educación sanitaria recibida por el sujeto y la existencia de aislamiento social/ambiente socioeconómico desfavorecido.

El formulario de recogida de datos se creó para realizar el presente trabajo. Esta basado en otros modelos de formularios pero con algunas modificaciones. Hemos preferido utilizar un instrumento de recogida de datos sencillo que se adecue a los métodos diagnósticos de los que disponemos en la unidad.

\section{Tratamiento de los datos}

Para el tratamiento de los datos recolectados en el estudio se utilizo el software para análisis de datos SPSS versión 15.0 para Windows XP.

En la primera fase del tratamiento de los datos se estudiaron cada una de las variables. En la segunda fase se realizó el análisis estadístico descriptivo entre variables. En la tercera fase se analizó la asociación entre variables mediante el test de la ji-cuadrado.

De esta forma podremos conocer el numero de pacientes que presenta una característica determinada (déficit de cuidados, ausencia de pulso, presencia de enfermedad vascular... etc.) del total de los pacientes estudiados, y la prevalencia de cada factor de riesgo. Además estudiamos la relación que hay entre diferentes variables o factores de riesgo mediante la ji-cuadrado.

Para valorar la existencia de enfermedad arterial periférica, utilizamos la presencia o ausencia de pulsos y el ITB y para valorar la existencia de pie diabético valoramos la alteración de la sensibilidad. A partir de la información recogida en la muestra para esta variable y de los valores obtenidos en los distintos factores de riesgo considerados en el estudio, se determinó la categorización del riesgo compuesta por los cuatro niveles anteriormente citados. 
La asociación entre variables mediante el test de jicuadrado nos aportó información relevante para nuestras conclusiones y para comparar con los resultados de otros estudios.

\section{Resultados}

La muestra estudiada ha estado compuesta por un total de 51 pacientes, procedentes de la unidad de diálisis de Segovia, entre los cuales hay 23 mujeres $(45,01 \%)$ y 28 hombres (54.9\%). La media de edad es de 69,37 años, con una desviación típica de 11,64 años, siendo la edad minima de 33 años y la máxima de 85 años. El tiempo medio en programa de diálisis es 5,16 años siendo el tiempo mínimo 1 mes y el máximo 20 años. En cuanto a las causas de la IRC, la diabetes es la primera causa con un $35,3 \%$, le siguen las glomerulonefritis con un $19,6 \%$, las causa desconocidas ocupan el $14,7 \%$, la enfermedad vascular es responsable de un 9,8\% de las causas de IR y en menor porcentaje están la enfermedad renal quística y las disproteinemias. Hay un $7,8 \%$ de otras causas.

En la Tabla 1 se muestran los porcentajes obtenidos para cada una de las variables definidas.

A la vista de los resultados comprobamos que la enfermedad cardiovascular es el factor de riesgo presente en mayor numero de pacientes, aparece en 29 sujetos lo que supone un $56,9 \%$.El porcentaje de diabéticos en nuestra Unidad, es de un 48\% (24 casos) de los cuales el 79,3\% hace mas de 10 años que es diabético y en y el $20,7 \%$ (6 casos) la evolución de esta enfermedad es menor de 10 años. Asimismo, otros factores destacables son la disminución o perdida de sensibilidad (30 casos; 58,8\%), la presencia de deformidades ( 29 casos; $56,9 \%$ ), el calzado inadecuado ( 28 casos; $54,9 \%$ ) y el déficit de cuidados de los pies con 23 casos lo que supone un 45,1\%.

El resto de los factores de riesgo aparecen en menor porcentaje: la presencia de helomas aparece en 11 sujetos $(21,6 \%)$, en 10 sujetos existía ulcera previa $(19,6 \%), 2$ pacientes tenían una amputación previa $(3,9 \%)$ y en 8 casos $(15,7 \%)$ se detectó aislamiento social o ambiente socioeconómico desfavorecido.

La prevalencia de arteriopatía periférica en nuestros pacientes se evalúa mediante la ausencia de pulsos.
De los 51 sujetos, 23 tienen pulsos ausentes por lo que la prevalencia de arteriopatía en nuestra unidad supone un $41,5 \%$.

\begin{tabular}{|c|c|c|c|}
\hline \multicolumn{2}{|l|}{ VARIABLES } & \multirow{2}{*}{$\begin{array}{c}\mathbf{n} \\
22 \\
29\end{array}$} & \multirow{2}{*}{$\begin{array}{c}\% \\
43,1 \\
56,9\end{array}$} \\
\hline Enf. cardiovascular & $\begin{array}{l}\text { No } \\
\text { Si }\end{array}$ & & \\
\hline Diabetes & $\begin{array}{l}\text { No } \\
\text { Si }\end{array}$ & $\begin{array}{l}26 \\
24\end{array}$ & $\begin{array}{l}52 \\
48\end{array}$ \\
\hline Duración diabetes & $\begin{array}{l}>10 \text { años } \\
<10 \text { años }\end{array}$ & $\begin{array}{c}23 \\
6\end{array}$ & $\begin{array}{l}79,3 \\
20,7\end{array}$ \\
\hline Pulsos & $\begin{array}{l}\text { ausentes } \\
\text { presentes }\end{array}$ & $\begin{array}{l}23 \\
28\end{array}$ & $\begin{array}{l}45,1 \\
54,9\end{array}$ \\
\hline Indice Tobillo Brazo & $\begin{array}{c}\text { Normal } \\
\text { Arteriopatía } \\
\text { Calcificación }\end{array}$ & $\begin{array}{l}25 \\
18 \\
8\end{array}$ & $\begin{array}{l}49,0 \\
35,3 \\
15,7\end{array}$ \\
\hline Déficit cuidados & $\begin{array}{l}\text { No } \\
\text { Si }\end{array}$ & $\begin{array}{l}28 \\
23\end{array}$ & $\begin{array}{l}54.9 \\
45.1\end{array}$ \\
\hline Mal Ambiente SE & $\begin{array}{l}\text { No } \\
\text { Si }\end{array}$ & $\begin{array}{c}43 \\
8\end{array}$ & $\begin{array}{l}84,3 \\
15,7\end{array}$ \\
\hline Amputación previa & $\begin{array}{l}\text { No } \\
\text { Si }\end{array}$ & $\begin{array}{c}49 \\
2\end{array}$ & $\begin{array}{l}96,1 \\
3,9\end{array}$ \\
\hline Úlcera previa & $\begin{array}{l}\text { No } \\
\text { Si }\end{array}$ & $\begin{array}{l}41 \\
10\end{array}$ & $\begin{array}{l}80,4 \\
19,6\end{array}$ \\
\hline Dism. sensibilidad & $\begin{array}{l}\text { No } \\
\text { Si }\end{array}$ & $\begin{array}{l}21 \\
30\end{array}$ & $\begin{array}{l}41,2 \\
58,8\end{array}$ \\
\hline Deformidades & $\begin{array}{l}\text { No } \\
\text { Si }\end{array}$ & $\begin{array}{l}22 \\
29\end{array}$ & $\begin{array}{l}43,1 \\
56,9\end{array}$ \\
\hline $\begin{array}{c}\text { Helomas/ } \\
\text { hiperqueratosis }\end{array}$ & $\begin{array}{l}\text { No } \\
\text { Si }\end{array}$ & $\begin{array}{l}40 \\
11\end{array}$ & $\begin{array}{l}78,4 \\
21,6\end{array}$ \\
\hline Calzado inadecuado & $\begin{array}{l}\text { No } \\
\mathrm{Si}\end{array}$ & $\begin{array}{l}23 \\
28\end{array}$ & $\begin{array}{l}45,1 \\
54,9\end{array}$ \\
\hline
\end{tabular}

Tabla 1. Variables

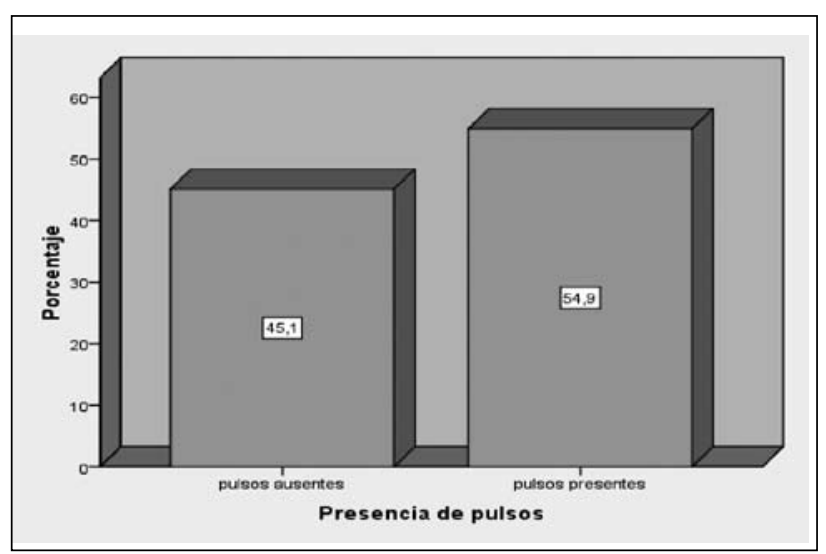

La valoración del estado vascular se completo con la determinación del Índice Tobillo Brazo que nos permite valorar la insuficiencia vascular en las extremidades inferiores. Los resultados que nos aporta esta variables 
son, que casi la mitad de nuestros pacientes (25) que corresponde a un $49 \%$ tienen un índice T/B normal, 18 sujetos, $(35,3 \%)$ presentan arteriopatía y $8(15,7)$ sujetos presentan alteración en este índice por calcificación arterial.

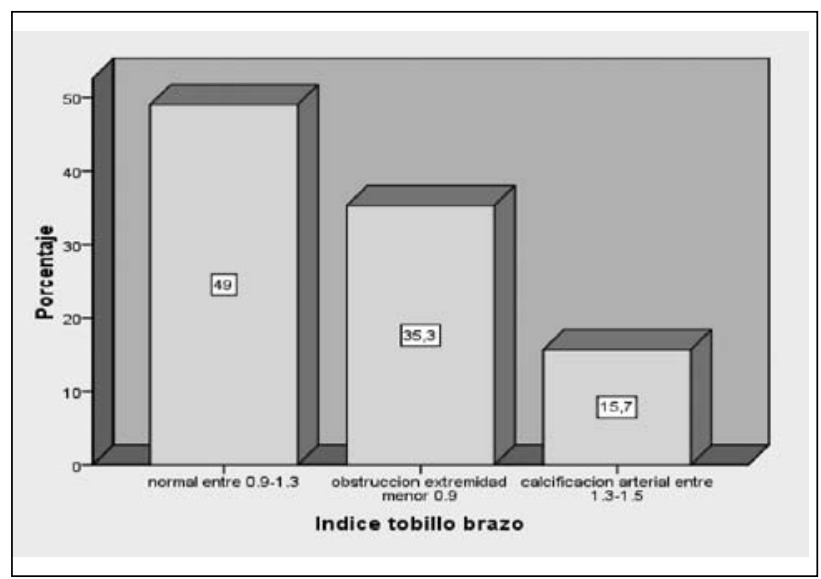

Para estudiar la presencia de neuropatía (pie diabético) revisamos los resultados que hemos obtenido en la variable: alteración de la sensibilidad. La disminución 0 pérdida de sensibilidad nos informa del riesgo de lesión que tiene un paciente. Encontramos que el 58,8\% (30 sujetos) tiene la sensibilidad alterada.

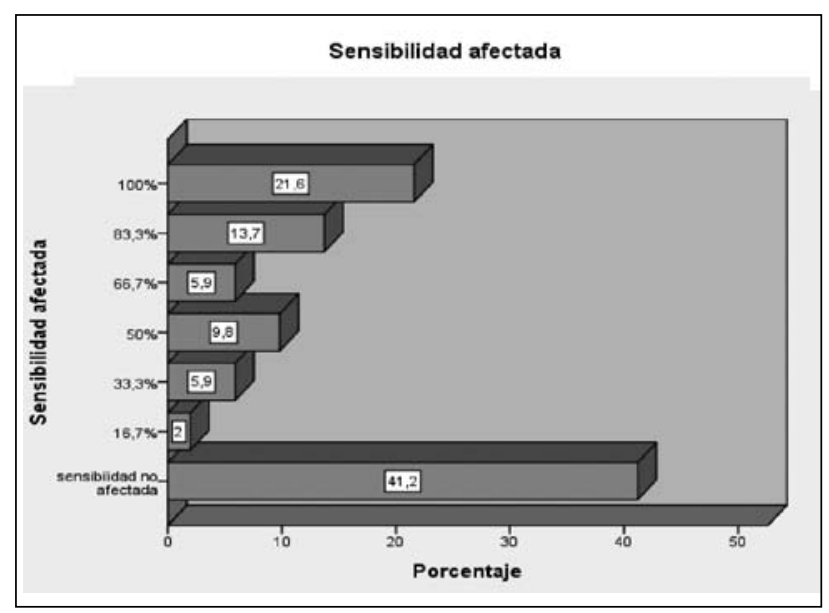

En cuanto a la categorización del riesgo los resultados obtenidos son: un $27,45 \%$ (14 sujetos) fue considerado en la categoría de riesgo nivel 1 el $25,49 \%$ ( 13 sujetos) se incluye en la categoría de riesgo 2; el 31,37\% (16 sujetos) pertenece al nivel de riesgo 3 y el $15,69 \%$ (8 sujetos) tiene un nivel de riesgo 4.

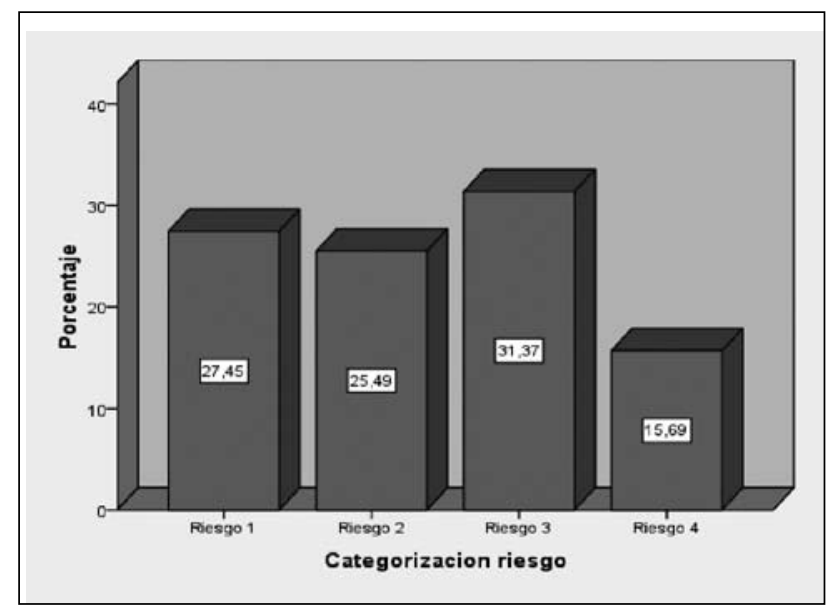

\section{Estudio de la asociación entre las variables consi- deradas}

Para analizar la asociación entre variables se ha utilizado, el test de la ji-cuadrado y el test de Anova. Para ambos contrastes se considera que existe una asociación significativa si el p obtenido resulta inferior a 0.05.

Del estudio de la asociación entre variables podemos destacar:

Los pacientes con arteriopatía periférica (EAP) son los diabéticos y los que presentan alguna enfermedad cardiovascular asociada a su Insuficiencia Renal. La prevalencia es mayor en los pacientes diabéticos que en los que no lo son pero tienen alteración cardiovascular. La diabetes también se asocia con la presencia de enfermedad cardiovascular.

La diabetes y la duración de esta, tienen influencia sobre la alteración de la sensibilidad protectora., los pacientes con mayor duración de la diabetes tienen mas frecuentemente alterada la sensibilidad protectora.

La alteración de la sensibilidad esta relacionada con el desarrollo de ulceras en extremidades inferiores .No encontramos asociación significativa entre ulcera y diabetes sin embargo de todos los pacientes que presentan ulceras, los pacientes diabéticos están en mayor porcentaje.

En la categorización de riesgo de los pacientes observamos que el mayor número de pacientes con riesgo muy elevado (riesgo 3) corresponde a los que tienen arteriopatía. Sin embargo no encontramos asociación 
entre la duración de la diabetes y la categorización del riesgo como establecen otros autores.

En cuanto al déficit de cuidados, en nuestro estudio se demuestra que esta relacionado con la aparición de ulceras en extremidades inferiores y que las personas con problemas de aislamiento social también presentan mayor déficit de autocuidados.

No hemos encontrado relación entre déficit de cuidados y presencia de helomas, deformidades en los pies, calzado inadecuado o sexo.

Un porcentaje elevado de nuestros pacientes con deformidades en los pies también presenta helomas.

\section{Discusión}

Los datos demográficos y clínicos de la población estudiada comparados con otros trabajos realizados con pacientes en diálisis ${ }^{(7)}$, difieren en la edad media (nuestros pacientes son más mayores), llevan mas tiempo en diálisis y tenemos más pacientes con diabetes como causa de la insuficiencia renal crónica. El $48 \%$ de la población es diabética frente a $23,3 \%$ de Mendias ${ }^{(8)}$ y el $27,05 \%$ de Lancho Casares $^{(7)}$.

La presencia de enfermedad cardiovascular tiene un porcentaje alto $(56,8 \%)$ y es semejante a los datos que aportan otros autores ${ }^{(7)}$.

La prevalencia de arteriopatía entre los pacientes de nuestro estudio es de un $45 \%$ que coincide con los datos de Bernal Pedreño(4), que tiene un 46,7\%.

El Índice tobillo/brazo nos muestra la presencia de arteriopatía en un $35,3 \%$ frente a un $17,9 \%$ que muestra Mendias Benítez ${ }^{(8)}$, este porcentaje es similar al numero de pacientes que tenemos con riesgo 3 es decir con alteración vascular y/o alteración de la sensibilidad.

Nuestro datos también se asemejan a los de Sánchez Perales ${ }^{(1)}$ en cuanto a que la arteriopatía es mas prevalente en diabéticos, sus datos dicen que en un $45 \%$ y en nuestro estudio este porcentaje es del $66,7 \%$ también encontramos asociación entre arteriopatía y enfermedad cardiovascular, el $78,3 \%$ de nuestros pacientes con arteriopatía tiene además enfermedades cardiovasculares.
En lo que respecta a la alteración de la sensibilidad, los datos de Bernal ${ }^{(4)}$ son que un 34,5\% tiene alterada la sensibilidad, mientras que en nuestros pacientes hay alteración de la sensibilidad en un 58,8\%. Esta diferencia puede deberse al numero de pacientes estudiado y/o al método aplicado. Hay autores que muestran que la no estandarización de métodos y la definición de umbral de sensibilidad puede dar diferentes resultados ${ }^{(12)}$.

Sin embargo otros autores afirman que la utilización de tres puntos es adecuada para detectar de forma temprana la neuropatía ${ }^{(13)}$.

Los pacientes de nuestra unidad que presentan ulceras en las extremidades inferiores, tienen todos alterada la sensibilidad. Esta relación entre neuropatía y complicaciones vasculares (úlceras) la citan también otros autores ${ }^{(14)}$ y en algunos estudios hechos con pacientes en diálisis peritoneal encuentran relación entre estos dos factores y la presencia de hipoalbuminemia ${ }^{(15)}$.

Los pacientes con alto riesgo de lesión (riesgo 3) detectados en nuestra unidad concuerdan con los datos de otras unidades de diálisis ${ }^{(4)}$.

Las deformidades de los pies, pueden influir en la aparición de ulceras y amputaciones. Nuestros resultados $(57 \%)$ están en consonancia con otros estudios ${ }^{(7)}$ realizados en pacientes en diálisis. Sin embargo en el momento del estudio la presencia de ulceras en nuestra Unidad es muy baja comparada con otras unidades.

El estudio de las repercusiones de déficit de cuidados, deformidades 0 alteraciones ortopédicas, calzado inadecuado y ambiente socioeconómico desfavorecido, aparece fundamentalmente en estudios hechos en Atención Primaria ${ }^{(17)}$ y en los Documentos de Consenso Ulceras y Pie diabético, tan solo hemos encontrado un estudio en el que se valoren alguno de estos factores en pacientes en diálisis ${ }^{(7)}$. La relación de estos factores de riesgo con la arteriopatía y el pie diabético es significativa y el numero de pacientes con estos factores se aproxima a los datos obtenidos en nuestro trabajo.

Con respecto a la presencia de exploración previa y protocolos de cuidados para este tipo de problemas, encontramos que los estudios realizados en Atención primaria, muestran que en este nivel de asistencia se interesan por este tipo de atención de enfermería ${ }^{(17)}$. 
En pacientes en diálisis solo hemos encontrado referencias de la existencia o no de exploración previa, en el trabajo de Bernal ${ }^{(4)}$. En nuestra unidad nunca habíamos realizado una valoración ni exploración completa a nuestros pacientes. En nuestros registros de enfermería se recogen datos referentes a algunos signos de arteriopatía y se describen lesiones y cuidados en la hoja de evolución y en el Plan de cuidados. Esta misma forma de trabajo la vemos reflejada en un trabajo publicado con pacientes en diálisis ${ }^{(7)}$. Hemos encontrado estudios realizados fuera de nuestro país que afirman que la revisión e intervención temprana en pacientes con estas patologías disminuye la tasa de complicaciones en las extremidades de los enfermos en diálisis ${ }^{(14,16)}$.

\section{Conclusiones}

Los resultados de nuestro estudio demuestran que un numero importante de nuestros pacientes en diálisis, presentan alteraciones vasculares y neuropáticas que son responsables de complicaciones muy importantes en los pies y las extremidades inferiores.

También podemos concluir a la vista de los datos que hay un numero importante de factores de riesgo que favorecen este tipo de lesiones y sobre los que se puede actuar para promover educación sanitaria y cuidados que disminuyan la aparición de este tipo de complicaciones.

La categorización de nuestros pacientes según su nivel de riesgo, nos permitirá establecer valoraciones y Planes de Cuidados específicos para actuar sobre estos problemas que presentan una alta prevalencia entre la población en diálisis.

La complejidad de la Insuficiencia Renal terminal y las tecnologías aplicadas en el tratamiento renal sustitutivo demandan muchos cuidados y energía por parte de la enfermera de diálisis, y en ocasiones no se presta la atención adecuada al cuidado de los pies en todas sus dimensiones.

Los resultados anteriormente expuestos nos demuestran que una exploración sistemática y protocolizada del estado vascular, neurológico y de autocuidados de nuestros pacientes, puede prevenir las ulceras y amputaciones de los pacientes renales.
Recibido: 11 Noviembre 2012

Revisado: 15 Noviembre 2012

Modificado: 20 Noviembre 2012

Aceptado: 22 Noviembre 2012

\section{Bibliografía}

1. N1- M. Á. Goicoechea: Enfermedad cardiovascular en pacientes con estadios 4 y 5 de enfermedad renal crónica. Pacientes en diálisis crónica. Nefrología 2004; 24 (Supl 6): 142-160.

2. Sánchez Perales $M C$, García Cortés $M J$, Borrego Utiel FJ, Viedma G, Gil JM, Pérez del Barrio $P$ et al. Incidencia y factores de riesgo de amputación no traumática de miembros inferiores en los pacientes en hemodiálisis. Nefrología 2005; 25(4): 399-405.

3. Deanna Holtman, NP; Gahtan Vivian MD. Peripheral Arterial Perfusion: Is it Adequate for Wound Healing? [Internet] Publicado 2008 [acceso 10 de marzo de 2011]. Disponible en http://www.medscape.com/viewarticle/579994.

4. E. Bernal Pedreño, E. Salces Sáez, A. Sambruno Giraldez. Exploración del pie a los pacientes diabéticos de una unidad de hemodiálisis. Rev. Soc. Esp. Enferm Nefrol 2009; 12 (2):111-116.

5. R. Marín, M.A Goicoechea, M. Gorostidi; A. Cases, J. Diez, G. Escolar, et al. Guía de la Sociedad Española de Nefrología sobre riñón y enfermedad cardiovascular. Nefrología 2006; 26(1): 41.

6. R. Aguila Márquez, M. Marquina Ramírez. Estado actual de la enfermedad arterial periférica oclusiva (EAP0). Acta Médica Grupo Ángeles.2007. Volumen 5. $\mathrm{N}^{\circ}$ : 187-196.

7. J.M. Lancho Casares, T. Juan Larma, C. de Vega Jiménez, M Nieto Guerrero, J. Acedo Agundez. Pacientes en programa de diálisis y arteriopatía periférica. Rev Soc Esp Enferm Nefrol. 2008; 11 (2):82-87. 
8. C Mendias Abujas A. Valoración del riesgo cardiovascular de pacientes de hemodiálisis. Rev Soc Esp Enferm Nefrol 2010; 13 (2): 127-132.

9. Grupo de trabajo sobre Ulceras Vasculares de la Asociación Española de Enfermería Vascular. Consenso sobre ulceras arteriales y pie diabético. Guía de práctica clínica. Asociación Española de Enfermería vascular. 2005.

10. Villa Estébanez R, Rodrigo Pendás JA, Veiras del Río 0. Arteriopatía periférica. [Internet]. En Fisterra.com. 11/05/2007 - Guías Clínicas 2007; 7 (42). [Acceso 19/4/11] disponible en http://www. fisterra.com/guias2/eap.asp\#mismo.

11. Grupo de Trabajo Internacional sobre el pie diabético. Consenso Internacional sobre el pie diabético. Madrid. Gramar. AG. 2001.

12. Dros J, Wewerinke $A$, Patrick $B, C$ van Weert $H$. Precisión de la prueba del monofilamento de diagnosticar la neuropatía periférica: una revisión sistemática. Ann Fam Med 2009; 7 (6):555558.
13. Feng $Y$, Schlösser FJ, Sumpio SER .EI monofilamento Semmes. Weinstein como instrumento de cribado para la neuropatía diabética periférica. Vasc Surg J. 2009; 50 (3):675-82.

14. Pliakogiannis $T$, Bailey, $S$ Cherukuri $M$, Ahmad, $T$, Oliver, Bargman JM, et al. Complicaciones vasculares de las extremidades inferiores en pacientes diabéticos en diálisis peritoneal. Clin Nephrol, 2008; 69 (5):361-7.

15. Lipscombe J,SV Jassal,S Bailey, JM Bargman, S Vas,Oreopoulos DG. Los podólogos pueden evitar amputaciones en pacientes diabéticos en diálisis peritoneal. Int. Dial Perit 2003; 23 (3):255-9.

16. Trilla Soler, $M$; Espluga Capdevila, $A$; Mengual Miralles, N;Bundó Vidiella, M; Juanola Costa, J; Aubà Llambrich, J; et al. Autocuidado y factores de riesgo de pie diabético en pacientes con diabetes mellitus tipo II. Aten Primaria, 1997; 20(4):186-90.

17. Richbourg M.J. Prevención de amputaciones en pacientes con enfermedad renal en estadio terminal. ¿Qué ocurre con el cuidado de los pies? EDTNA ERCA JOURNAL 1998. 24(4): 4-10. 